\title{
Long-term renal function and continence status in patients with cloacal malformation
}

\author{
Luis H.P. Braga, MD; Armando J. Lorenzo, MD; Sumit Dave, MD; Maria H. Del-Valle, MD; Antoine E. Khoury, MD; \\ Joao L. Pippi-Salle, MD, PhD
}

\begin{abstract}
Introduction: Urinary continence after cloacal repair is difficult to achieve and renal outcome in patients with cloacal malformations has been scarcely reported. As a result, we reviewed our experience with cloacal malformations to determine the status of continence and the long-term renal function in these children.
\end{abstract}

\begin{abstract}
Methods: A retrospective chart review from 1990 to 2003 identified 12 patients with cloacal malformation ( 1 posterior, 4 complex and 7 classical) who underwent surgical reconstruction. The confluence was defined as high (common channel $\geq 3 \mathrm{~cm}$ ) and low $(<3 \mathrm{~cm})$ by cystovaginoscopy. Renal ultrasound, voiding cystouretrogram, renal scan and sacral radiograph were performed in all children. Most patients underwent 1-stage abdominoperineal pull-through, applying the principle of total urogenital sinus mobilization. We collected data regarding hydronephrosis, vesicoureteral reflux and split differential renal function. Renal outcome was evaluated based on glomerular filtration rate and ageadjusted serum creatinine values $(\mu \mathrm{mol} / \mathrm{L})$. Urinary continence was defined as a dry interval $>4$ hours.
\end{abstract}

Results: Patients' mean age at surgery was 20 months (range 7-29 mo). Of the 12 children who underwent cloacal repair, 7 (58.3\%) had a common channel $\geq 3 \mathrm{~cm}$. Renal anomalies were identified in 3 of $12(25 \%)$ girls: there were 2 solitary kidneys and 1 pelvic kidney. Lumbar-sacral radiography demonstrated bony abnormalities in 11 of the 12 (91.6\%) cases: hemivertebra in 3 cases, sacral agenesis in 4 cases, hypoplastic sacrum in 3 cases and bifid sacrum in 1 case. Total urogenital sinus mobilization through an abdominoperineal approach in a single stage was performed in 8 girls. Follow-up ranged from 4 to 14 years (mean $8.5 \mathrm{yr}$ ). Eight $(66.6 \%)$ children had dry intervals $>4$ hours, $5(62.5 \%)$ of them were on clean intermittent catheterization through a Mitrofanoff channel and $1(12.5 \%)$ was through the urethra. The remaining $2(25 \%)$ patients were voiding spontaneously. Three $(33.3 \%)$ patients were totally incontinent, and $1(8.3 \%)$ patient was awaiting reconstruction. The mean measured glomerular filtration rate was $93.5 \mathrm{~mL} / \mathrm{min} / 1.73 \mathrm{~m}^{2}$ (range $34-152 \mathrm{~mL} / \mathrm{min} / 1.73 \mathrm{~m}^{2}$ ). Four $(57.1 \%$ ) of 7 patients who had a common channel $\geq 3 \mathrm{~cm}$ ended up needing augmentation cystoplasty, compared with none of the patients with a common channel $<3 \mathrm{~cm}(57.1 \%$ v. $0 \%, p=0.038)$.

Conclusion: Urinary continence can be achieved in most patients with cloacal malformation at the expense of major reconstructive surgery and despite the presence of associated urological abnormalities. However, these children harbour an important risk for renal impairment later in life and should be closely monitored.

CUAJ 2007;1(4):371-6

\section{Introduction}

Persistent cloaca is a rare female malformation in which the rectum, vagina and urethra fuse together to form a common channel that opens in the perineum as a single orifice. It is a severe congenital anomaly that occurs with an incidence of 1 in $50000^{1}$ and includes a wide spectrum of complex anatomic abnormalities ranging from low defects with normal urinary tract to severe pelvic malformations associated with multiple uro-gynecological abnormalities.

Urinary continence after cloacal repair is difficult to achieve and is obtained in many only after several surgical interventions., ${ }^{2,3}$ Further, associated congenital anomalies such as tethered cord and renal malformations are common, adversely affecting the achievement of continence as well as the ultimate renal function. ${ }^{4}$ Renal impairment, associated with severe morbidity and mortality, leads to chronic renal failure in about one-half of the patients with cloacal malformations. ${ }^{5}$ To date, renal outcomes after cloacal repair have been scarcely reported. ${ }^{5}$ Taking these issues into account, we sought to review our experience with cloacal malformations over the past 14 years to determine the continence status and long-term renal function of these children.

\section{Methods}

The study was approved by our institution's Research Ethics Board. A retrospective chart review from January 1990 to December 2003 identified 12 patients with cloacal malformations who underwent surgical reconstruction. These cloacal anomalies were classified as 3 types: classical, posterior and complex. A classical cloacal malformation is defined by 
the presence of a common channel draining the bladder, vagina and rectum, which then opens into a single orifice in the perineum (Fig. 1). In contrast, posterior cloacal malformation patients have the genitourinary common channel deviated posteriorly, entering the anterior rectal wall. Complex cloacal malformation is considered when the common channel is longer than $3 \mathrm{~cm}$ and other mullerian abnormalities, such as uterus didelphus, 2 hemivaginas or atretic mullerian structures, are present. Our series comprised 1 posterior, 4 complex and 7 classical cloacal malformations. The level of confluence was determined by either cystovaginoscopy (with direct measurement of the common channel distance from the perineum) or by cystogram and distal colostogram (Fig. 2). Following Pena and colleagues' classification, a high confluence was characterized by a channel length $\geq 3 \mathrm{~cm}^{6}$

All patients underwent renal and pelvic ultrasound, voiding cystouretrogram (VCUG) and renal scan before surgery. Lumbar-sacral radiographs were obtained before the reconstruction to identify bony abnormalities such as hemivertebra, hypoplastic sacrum or sacral agenesis. Since 1998, spinal ultrasound has been performed in patients less than 3 months of age to screen for associated low conus or occult spinal dysraphism, reserving the use of MRI for those with poor visualization on ultrasound, ossification precluding ultrasound evaluation or with an abnormal spinal

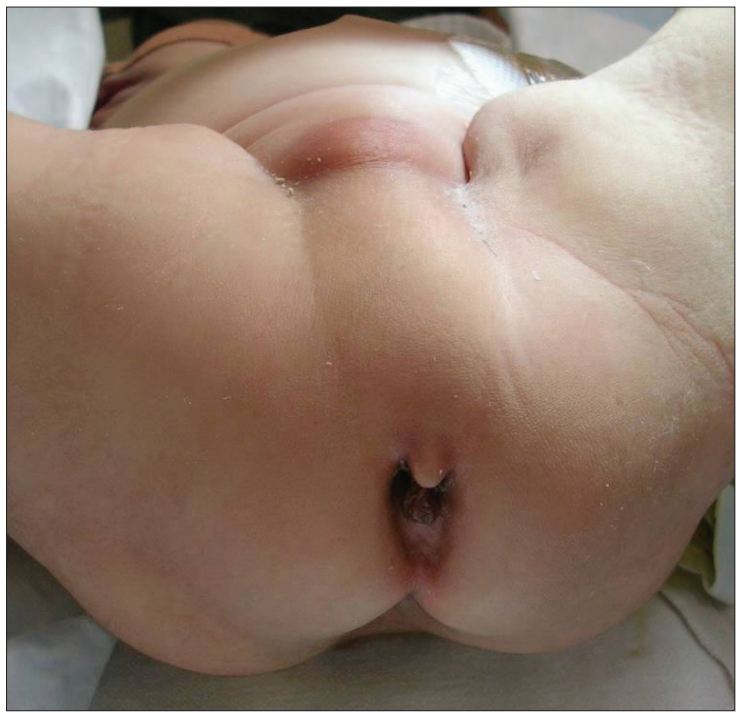

Fig. 1. This photograph shows a cloacal malformation, with a single opening seen at the perineum. ultrasound. Occult spinal dysraphism was suspected when the spinal cord resided below the level of the second lumbar vertebra on spinal ultrasound, if the filum terminale was diffusely thickened or if the cord appeared to be fixed to a spinal lipoma. ${ }^{7}$

Early in our series, 4 patients underwent a 2stage procedure in which the rectal component was repaired first, leaving the urogenital sinus for a secondary operation. In the remaining 8 girls, a 1-stage abdomino-perineal pull-through was performed. Following the principles originally described by Levitt and Pena, ${ }^{8}$ a total urogenital sinus mobilization allowed the urethra and vagina to be brought down as a single unit to the perineum, with the rectum positioned posteriorly between the levator ani muscles as a separate structure. The abdominal part of this approach was used in cases of complex cloacal malformations to deal with atretic mullerian structures or with duplication anomalies.

We collected data regarding pre- and postoperative hydronephrosis, vesicoureteral reflux (VUR) and split differential renal function. Renal outcome was evaluated based on glomerular filtration rate (GFR) and age-adjusted serum creatinine values ( $\mu \mathrm{mol} / \mathrm{L})$. GFR was measured by plasma creatinineethylenediaminetetraacetic acid and corrected to $1.73 \mathrm{~m}^{2}$ body surface area. Urinary continence

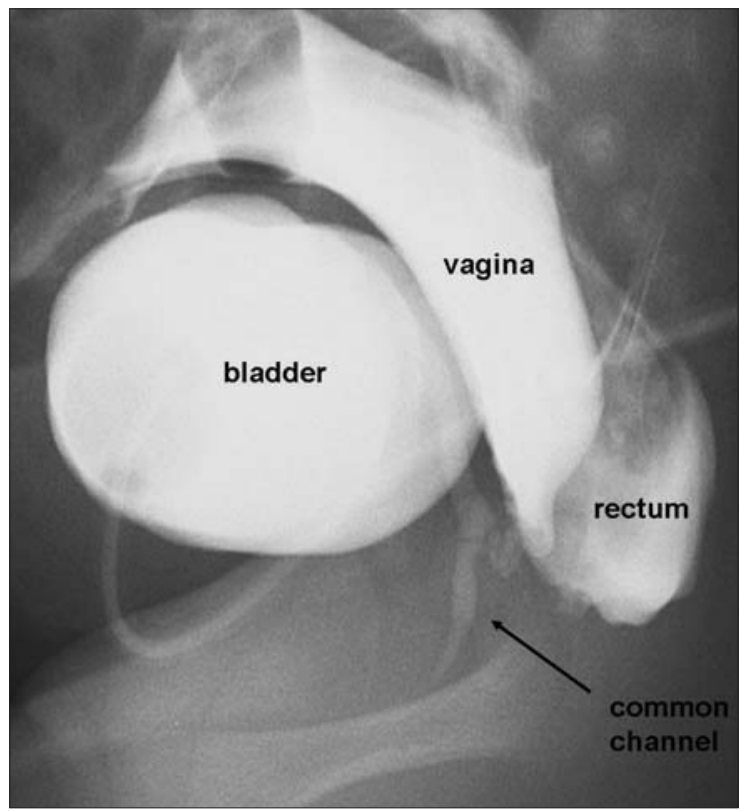

Fig. 2. This genitogram performed during the first week of the patient's life shows the rectum, vagina and bladder fusing into a common channel that opens in the perineum as a single orifice. 
was defined as dry intervals greater than 4 hours (totally continent). Patients unable to hold urine for at least 2 hours were considered incontinent.

We conducted descriptive analysis with the assistance of commercially available statistics software, SPSS version 11.0 (SPSS Inc., Chicago, III.) and considered $p<0.05$ statistically significant. We used Fischer's exact test for comparative analysis owing to the small size of our sample.

\section{Results}

Patients' mean age at surgery was 20 months (range $7-29 \mathrm{mo})$. Of the 12 children, 7 had a high defect $(58.3 \%)$ with a common channel $\geq 3 \mathrm{~cm}$, measured by preoperative cystovaginoscopy. Hydronephrosis was initially seen in 7 patients $(58.3 \%)$ and 5 of these (71.4\%) were found to have VUR. VCUG demonstrated grade II VUR in 2 children, grade III VUR in 2 children and grade IV VUR in 1 child. Reflux was bilateral in 3 and unilateral in 2 children, respectively. Of the 5 patients with VUR, 4 underwent intravesical ureteral reimplantation at the time of cloacal reconstruction. Three of 12 (25\%) girls had preoperative renal scarring or dysplasia with a split differential renal function of $12 \%, 26 \%$ and $44 \%$, respectively, in the affected kidneys. Renal anomalies were identified in 3 of the $12(25 \%)$ children: there were 2 solitary kidneys and 1 pelvic kidney. Table 1 summarizes the urological malformations seen in the 12 patients with cloacal anomalies.

Lumbar-sacral radiography demonstrated bony abnormalities in 11 of the 12 (91.6\%) cases: hemivertebra in 3 cases, sacral agenesis in 4 cases, 3 cases of hypoplastic sacrum and 1 case of bifid sacrum. Spinal ultrasound detected a low

Table 1: Urological malformations seen in the 12 patients with cloacal anomalies

\begin{tabular}{lc}
\hline Urological malformations & No. (and \%) of patients \\
\hline Hydronephrosis & $7(58.3)$ \\
Vesicoureteral reflux* & $5(41.6)$ \\
Unilateral & $2(16.6)$ \\
Bilateral & $3(25.0)$ \\
Renal scarring or dysplasia & $3(25.0)$ \\
Solitary kidney & $2(16.6)$ \\
Pelvic kidney & $1(8.3)$ \\
\hline *Voiding cystouretrogram of patients with vesicoureteral reflux (VUR) \\
demonstrated that 2 patients had grade II VUR, 2 patients had grade III \\
VUR and 1 patient had grade IV VUR.
\end{tabular}

conus (spinal cord below the level of L2) in 8 (75\%) children. MRI supported the diagnosis of tethered cord in $3(37.5 \%)$ of these 8 patients, all of whom underwent additional surgery for spinal cord untethering.

All children underwent a diverting colostomy in the first week of life. One infant girl had a vaginostomy draining urine at the time of the referral. No patients required a vesicostomy to decompress the urinary tract. Two of the most recent cases were managed with clean intermittent catheterization while waiting for the definitive surgical reconstruction. The surgical approach was prone posterior sagital in 4 patients and combined posterior sagital and supine in 8 patients. Total urogenital sinus mobilization through an abdominoperineal approach in a single stage was performed in 8 girls. The remaining 4 girls had initial rectal pull-through procedures followed by mobilization of the urogenital sinus at a second stage.

Follow-up ranged from 4 to 14 years (mean $8.5 \mathrm{yr}$ ). There was 1 late complication (8.3\%) due to bowel obstruction secondary to intra-abdominal adhesions. There were no cases of urethrovaginal fistulae. Bladder augmentation was performed in 4 of $12(33.3 \%)$ patients with complex cloacal malformations, including 2 ileocystoplasties, 1 colocystoplasty and 1 gastro-ileocystoplasty. These 4 girls had concomitant bladder neck closure and creation of a Mitrofanoff catheterizable channel. One patient developed bladder stones 5 years after bladder augmentation and required cystoscopy and lithotripsy through the catheterizable channel.

At most recent follow-up, 8 (66.6\%) children had dry intervals $>4$ hours, $5(62.5 \%)$ of them were on clean intermittent catheterization through a Mitrofanoff channel and $1(12.5 \%)$ through the urethra. The remaining 2 (25\%) were voiding per urethra. Four of the 6 patients who were on clean intermittent catheterization had a closed bladder neck. Three (33.3\%) patients had total urinary incontinence, and 1 child (8.3\%) is still awaiting reconstruction for treatment of urinary incontinence (Table 2).

At a mean age of 10.3 years, the average measured GFR was $93.5 \mathrm{~mL} / \mathrm{min} / 1.73 \mathrm{~m}^{2}$ (range 34-152 mL/min/1.73 m²). Evaluation of renal function showed that 9 of $12(75 \%)$ children had an abnormal GFR $(<80 \mathrm{~mL} / \mathrm{min})$. Three of 9 had a normal age-adjusted serum creatinine level despite 
a reduced GFR. Overall, 9 of 12 (75\%) girls had some degree of renal function impairment (Table 3).

We attempted to compare the length of cloacal defect (common channel $\geq 3 \mathrm{~cm}$ or not) with incidence of renal anomalies, presence of impaired renal function, need for bladder augmentation and urinary continence (Table 4). Renal anomalies were present in 2 of 7 patients with common channels $\geq 3 \mathrm{~cm}$, compared with 1 of 5 with common channels $<3 \mathrm{~cm}(22.8 \%$ v. $20 \%, p=0.73)$. Impaired renal function (GFR $<80 \mathrm{~mL} / \mathrm{min}$ ) occurred in 5 of 7 children who had long common channels, compared with 3 of 5 who had short common channels $(71.4 \%$ v. $60 \%, p=0.24)$. We found a statistically significant difference between long and short common channels with regard to the patient's need for bladder augmentation. Four of $7(57.1 \%)$ patients who had a common channel $\geq 3 \mathrm{~cm}$ ended up with an augmentation cystoplasty, compared with none of the patients who had a common channel $<3 \mathrm{~cm}$ $(57.1 \%$ v. $0 \%, p=0.038)$. Finally, we found a difference in urinary dryness when comparing long and short common channels $(85.7 \%$ v. $40 \%, p=$ 0.30). Girls with longer common channels had a higher rate of urinary dryness at the expense of a higher percentage of bladder augmentation and bladder neck closure.

\section{Discussion}

Urological problems after definitive repair of cloacal malformations in children include urinary incontinence, poor renal function and recurrent urinary tract infections. Urinary continence was achieved in $67 \%$ of the patients in this study, a rate

\begin{tabular}{lc}
$\begin{array}{l}\text { Table 2: Continence status of the } \mathbf{1 2} \text { patients with } \\
\text { cloacal malformation }\end{array}$ \\
\hline Continence status (dryness) & $\begin{array}{c}\text { No. (and \%) } \\
\text { of patients }\end{array}$ \\
\hline Continent (dry interval $>4 \mathrm{~h}$ ) & $8(66.6)$ \\
Incontinent or dry interval $<2 \mathrm{~h}$ & $3(25.0)$ \\
Awaiting surgery for urinary incontinence & $1(8.3)$ \\
Bladder emptying and continence & \\
$\quad$ Continent with CIC and bladder augmentation & $4(33.3)$ \\
Continent with CIC alone & $2(16.6)$ \\
Continent voiding per urethra & $2(16.6)$ \\
Incontinent voiding per urethra & $3(25.0)$ \\
\hline CIC = clean intermittent catheterization. & \\
\hline
\end{tabular}

comparable with other reports $(60 \%-83 \%)^{4,9}$ Despite this apparently high rate of urinary continence in complex cases, dryness was achieved at the expense of major reconstructive surgery, such as bladder augmentation with or without bladder neck closure and clean intermittent catheterization through a Mitrofanoff conduit. In the present study, $63 \%$ of children who had dry intervals $>4$ hours were on clean intermittent catheterization and $50 \%$ of them had an augmentation cystoplasty with bladder outlet closure and creation of a catheterizable conduit. Only $17 \%$ of girls who achieved dryness were voiding through the urethra. These results are far from ideal, but they are similar to those published in another series that reported that $46 \%$ of continent patients required major surgery and that no more than $22 \%$ voided spontaneously and were dry. ${ }^{4}$ Conversely, in Hendren ${ }^{10}$ and Pena' $\mathrm{s}^{11}$ series, $60 \%$ and $54 \%$ of children, respectively, voided spontaneously and were dry, but neither author mentioned the need for additional surgery at the level of the bladder neck.

Recently, Pena reported a continence rate of $28 \%$ in girls with common channels $>3 \mathrm{~cm}$ and showed that these patients had a high likelihood of needing clean intermittent catheterization, compared with those with short common channels. ${ }^{6}$ With that in mind, we attempted to compare dryness with length of common channel and we found that the longer the common channel, the higher the chance of being dry ( $85.7 \%$ v. $40 \%)$. At first impression, this seemed illogical as patients with shorter common channels had better anatomy and should have had more favourable outcomes as shown by Pena and colleagues $^{6}$ and Warne and coauthors. ${ }^{3}$ However, after careful analysis of our data, $60 \%$ of children with common channels $\geq 3 \mathrm{~cm}$ required bladder augmentation associated with bladder neck closure and Mitrofanoff conduit, compared with none of the patients who had common channels $<3 \mathrm{~cm}$;

$\begin{aligned} & \text { Table 3: Renal outcome of the } \mathbf{1 2} \text { patients } \\
& \text { with cloacal malformation at most recent } \\
& \text { follow-up (mean } \mathbf{8 . 5} \text { yr) }\end{aligned}$
\begin{tabular}{lc} 
Impaired renal function & $\begin{array}{c}\text { No. (and \%) } \\
\text { of patients }\end{array}$ \\
\hline GFR $<80 \mathrm{~mL} / \mathrm{min} / 1.73 \mathrm{~m}^{2}$ & $9(75.0)$ \\
High age-adjusted serum creatinine & $6(50.0)$ \\
Overall & $9(75.0)$ \\
GFR = glomerular filtration rate. &
\end{tabular}


Table 4: Comparison of type of cloacal defect with renal anomalies, impaired renal function, bladder augmentation and urinary incontinence

\begin{tabular}{lccc}
\hline & $\begin{array}{c}\text { No. (and \%) } \\
\text { of patients with long } \\
\text { common channels } \\
(\geq 3 \mathrm{~cm}) ; n=7\end{array}$ & $\begin{array}{c}\text { No. (and \%) of } \\
\text { patients with short } \\
\text { common channels } \\
(<3 \mathrm{~cm}) ; n=5\end{array}$ & $\begin{array}{c}\text { Fisher's exact test; * } \\
p \text { value }\end{array}$ \\
Variables & $2(28.6)$ & $1(20.0)$ & 0.73 \\
\hline Renal anomalies & $5(71.4)$ & $3(60.0)$ & 0.24 \\
Impaired renal function & $4(57.1)$ & $0(0.0)$ & 0.038 \\
Bladder augmentation & $6(85.7)$ & $2(40.0)$ & 0.30 \\
Urinary continence & & & \\
\hline *Used when the number of cells is less than 5. & & \\
\hline
\end{tabular}

this explains the high rate of dryness achieved after all these procedures.

Pena and colleagues have previously described that sacral bony abnormalities are associated with urinary incontinence, especially in patients with anorectal malformations. ${ }^{6}$ Warne and colleagues have shown that the incidence of bony abnormalities is higher in cloacal, compared with anorectal malformations. ${ }^{12}$ We could not correlate sacral anomalies to urinary continence as most of our patients (92\%) had some type of sacral bony abnormality. Despite this high association with sacral defects, only $37 \%$ of our patients had a spinal anomaly that required spinal cord untethering. Similar findings were reported by Hendren who found that $27 \%$ of his patients had associated tethered cord..$^{13}$

We acknowledge the methodologic constraints of a retrospective analysis and the small number of patients as important limitations of this study. Nonetheless, we speculate that the urinary incontinence seen in patients with cloacal malformations is multifactorial. It may be secondary to structural abnormalities of the bladder, bladder neck and urethra, sacral dysplasia or agenesis, and intrinsic or iatrogenic neurovesical dysfunction (surgical denervation). Previous reports suggest that $60 \%$ of cloacal malformation patients have an atonic type of neurogenic bladder, requiring clean intermittent catheterization to empty. In the remaining cases, urodynamic studies may help clarify the etiology of the ongoing incontinence.

There have been few reports of long-term renal function in children with cloacal malformations. ${ }^{5,9}$ Associated anomalies, such as solitary kidney, renal dysplasia, VUR and renal scarring, can negatively affect renal outcome. As shown by Warne and colleagues' experience, $25 \%$ of patients with a solitary kidney, $44 \%$ with renal dysplasia, $72 \%$ with VUR and $47 \%$ with renal scarring had some degree of renal function impairment. ${ }^{5}$ Accordingly, we found that $80 \%$ of our patients who had VUR and $100 \%$ of those who had renal anomalies had impaired renal function after a mean follow-up of 8.5 years. We also identified that girls with longer common channels $(\geq 3 \mathrm{~cm})$ had worse renal outcome, compared with those who had short common channels, but the difference was not significant. This trend may prove to be statistically significant if a larger cohort is investigated.

It was interesting to note that 3 children had normal serum age-adjusted creatinine values despite a reduced GFR. It is well known that abnormal serum creatinine is indicative of poor renal function, but these findings suggest that normal serum creatinine should not be used as a predictor of good renal function as it is not as sensitive as GFR. ${ }^{5}$

In summary, patients with cloacal malformations constitute a complex group of children in whom urinary dryness can be achieved at the expense of major reconstructive surgery and despite the presence of associated urological abnormalities. However, these patients harbour an important risk for renal impairment later in life and should be closely followed by pediatric nephrologists and urologists.

From the Division of Pediatric Urology, Hospital for Sick Children, University of Toronto, Toronto, Ont.

This article has been peer reviewed.

Competing interests: None declared.

References

1. Krstic ZD, Lukac M, Lukac R, et al. Surgical treatment of cloacal anomalies. Pediatr Surg Int 2001;17:329-33. 
2. Shimada K, Matsumoto F, Tohda A, et al. Urinary control after the definitive reconstruction of cloacal anomaly. Int J Urol 2005;12:631-6.

3. Warne SA, Wilcox DT, Ransley PG. Long-term urological outcome of patients presenting with persistent cloaca. J Urol 2002;168:1859-62.

4. Hendren WH. Urological aspects of cloacal malformations. J Urol 1988;140:1207-13.

5. Warne SA, Wilcox DT, Lederman SE, et al. Renal outcome in patients with cloaca. J Urol 2002; 168:2548-51.

6. Pena A, Levitt MA, Hong A, et al. Surgical management of cloacal malformations: a review of 339 patients. J Pediatr Surg 2004;39:470-9.

7. Mosiello G, Capitanucci ML, Gatti C, et al. How to investigate neurovesical dysfunction in children with anorectal malformations. J Urol 2003;170:1610-3.

8. Levitt MA, Pena A. Pitfalls in the management of newborns with cloacas. Pediatr Surg Int 2005;21:264-9.

9. Rink RC, Herdon A, Cain MP, et al. Upper and lower urinary tract outcome after sur- gical repair of cloacal malformations: a three-decade experience. BJU Intern, 2005:96:131-4.

10. Hendren WH. Cloaca, the most severe degree of imperforate anus. Ann Surg 1998:228:331-9.

11. Pena A. Anorectal malformations. Semin Pediatr Surg 1995;4:35-43

12. Warne SA, Godley ML, Wilcox DT. Surgical reconstruction of cloacal malformation can alter bladder function: a comparative study with anorectal anomalies. J Urol 2004; 172:2377-81

13. Hendren WH. Cloacal malformations: experience with 105 cases. J Pediatr Surg 1992;27:890-1.

Correspondence: Dr. J.L. Pippi-Salle, 555 University Ave., M299, Toronto ON M5G 1X8; pippi.salle@sickkids.ca

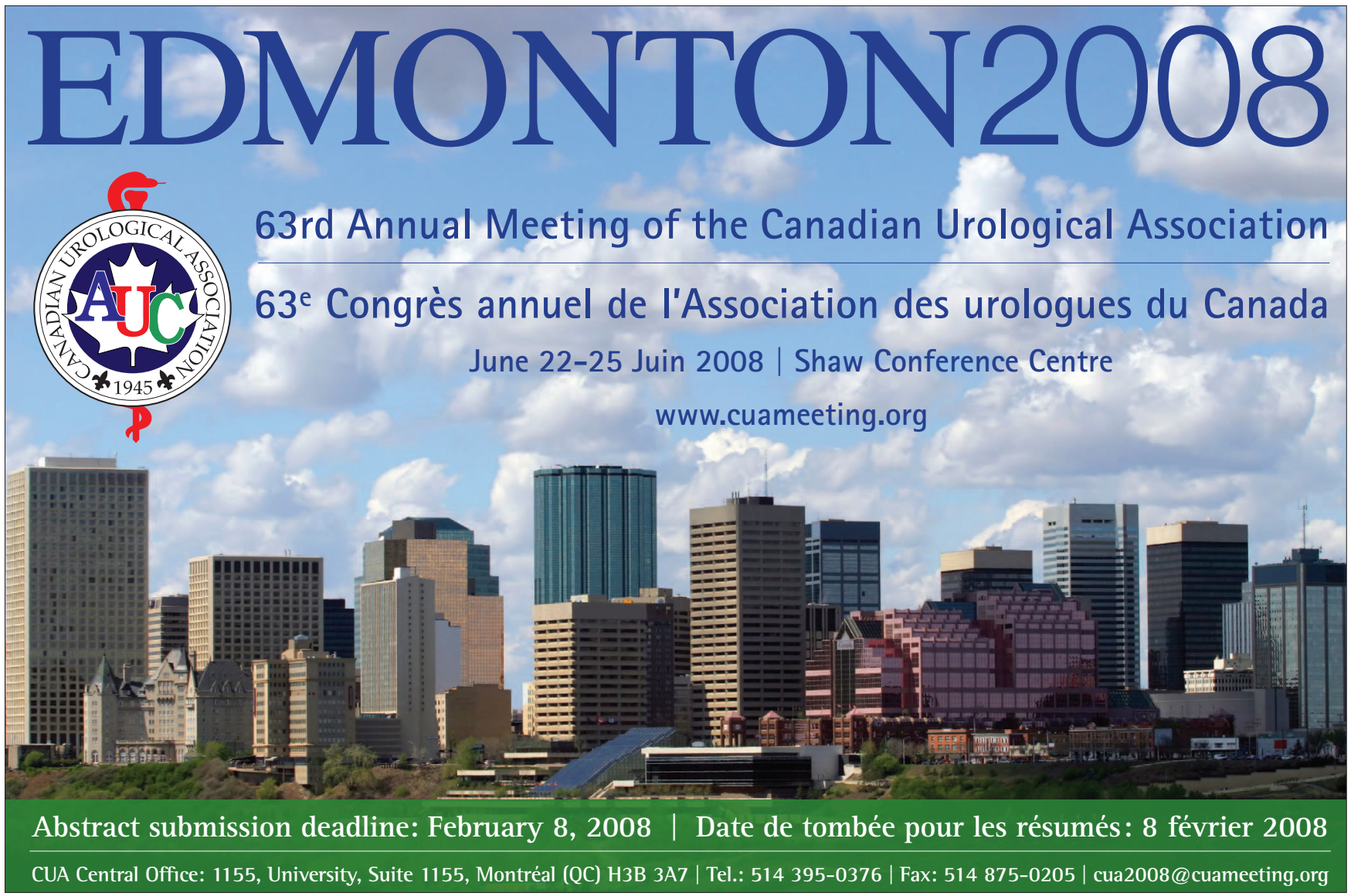

